\title{
New Insights into the Pathogenesis and Treatment Strategies in IgA Nephropathy
}

\author{
Katrin Scionti ${ }^{a} \quad K^{2}$ aren Molyneux ${ }^{b}$ Haresh Selvaskandan ${ }^{a, b}$ Jonathan Barratt ${ }^{a, b}$ \\ Chee Kay Cheung ${ }^{a, b}$ \\ aDepartment of Cardiovascular Sciences, University of Leicester, Leicester, UK; b John Walls Renal Unit, University \\ Hospitals of Leicester NHS Trust, Leicester, UK
}

\section{Keywords}

Immunoglobulin A nephropathy · Pathogenesis ·

Treatment

\begin{abstract}
Background: Immunoglobulin A nephropathy ( $\lg A N)$ is the most common form of primary glomerulonephritis worldwide. It is defined by mesangial IgA deposition, with consequent mesangial cell proliferation, inflammation, and tubulointerstitial fibrosis. Summary: Approximately 30\% of affected patients will progress to end-stage kidney disease within 20 years of diagnosis. Currently, there is no diseasespecific treatment available and management recommendations are, in general, limited to optimization of lifestyle measures and use of renin-angiotensin-aldosterone system blockers. More recently, advances in the understanding of the pathogenesis of IgAN have informed the development of novel therapeutic strategies that are now being tested in clinical trials. These have focused on different areas that include modulating the production of poorly galactosylated $\lg \mathrm{A} 1$, which is central to the development of $\lg \mathrm{AN}$, and inhibiting the downstream signaling pathways and complement activation that are triggered following mesangial IgA1 deposition. In this review, we will summarize important pathogenic mechanisms in lgAN and highlight important areas of
\end{abstract}

karger@karger.com www.karger.com/gdz

Karger $\stackrel{\text { ' }}{5}$
(C) 2021 The Author(s)

Published by S. Karger AG, Basel

This is an Open Access article licensed under the Creative Commons Attribution-NonCommercial-4.0 International License (CC BY-NC) (http://www.karger.com/Services/OpenAccessLicense), applicable to the online version of the article only. Usage and distribution for commercial purposes requires written permission. interest where treatment strategies are being developed. Key messages: IgAN is a common form of primary glomerulonephritis for which there is no current approved specific therapy. Recent advances in the understanding of its pathogenesis have led to the development of novel therapies, with the hope that new treatment options will be available soon to treat this condition.

(c) 2021 The Author(s)

Published by S. Karger AG, Basel

\section{Introduction}

Immunoglobulin A nephropathy (IgAN) is the most common primary glomerulonephritis worldwide, with an estimated incidence of at least 2.5 per 100,000 per year in adults, and a higher frequency in East Asian countries [1]. The condition is characterized by IgA deposition in the glomerular mesangium, which initiates inflammatory cytokine release and complement activation, resulting in mesangial cell proliferation, extracellular matrix deposition, tubulointerstitial fibrosis, and, in approximately $30 \%$ of patients, end-stage kidney disease within 20 years of diagnosis. The exact mechanisms that result in IgA deposition are not completely understood; however, the frequent recurrence of glomerular IgA deposition after kidney transplantation 
and reports of clearance of deposited IgA after transplantation of an affected kidney into a non-IgAN recipient indicate that the defect in IgAN is systemic rather than confined to the kidney $[2,3]$.
At present, there is no disease-specific treatment available for IgAN. Treatment recommendations are mainly limited to optimization of lifestyle measures, including strict blood pressure control, weight reduction, smoking

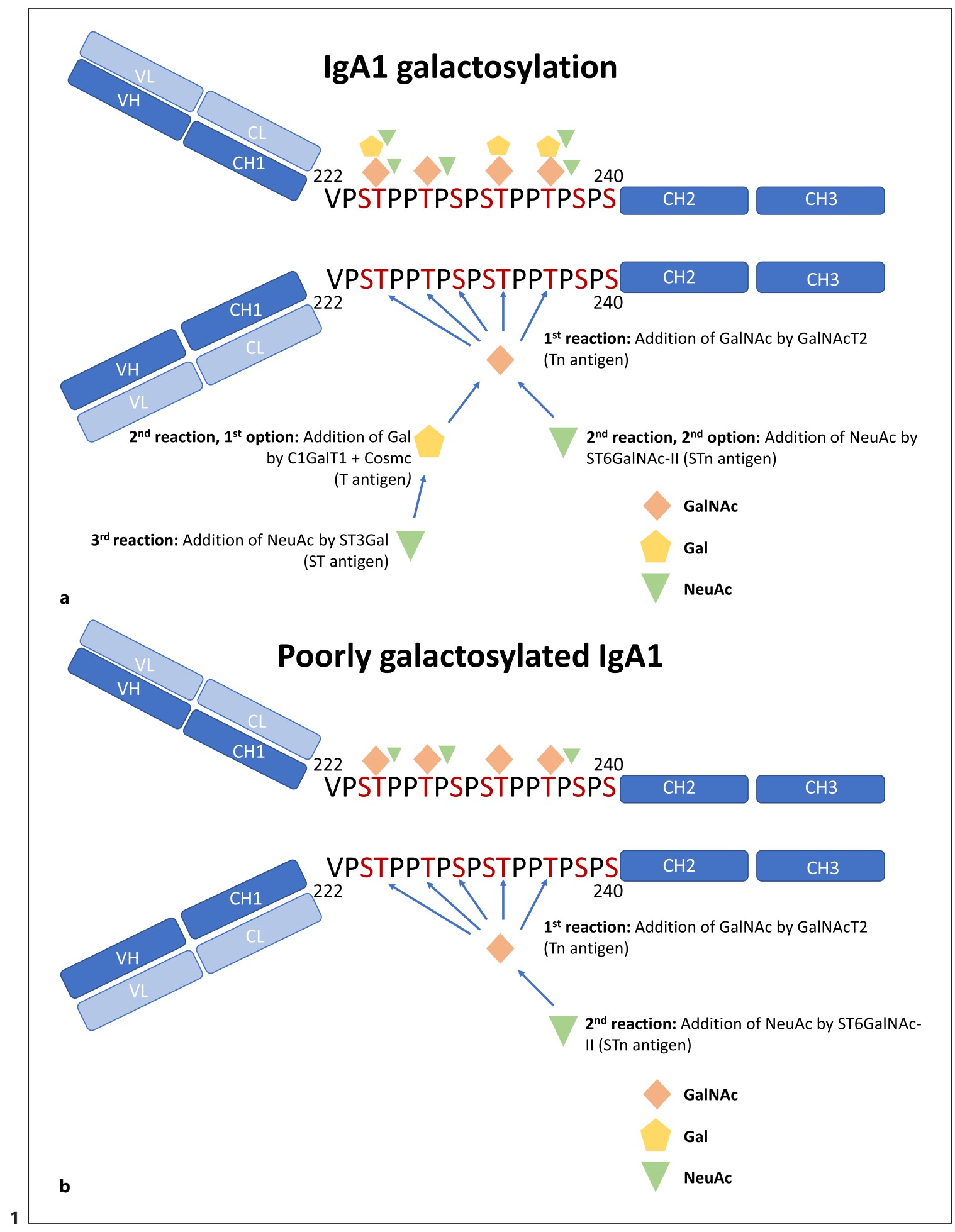

(For legend see next page.) 
cessation and cholesterol reduction, and use of renin-angiotensin-aldosterone system (RAAS) blockers. Current Kidney Disease: Improving Global Outcomes guidelines suggest the use of corticosteroids if these measures fail to reduce proteinuria [4]. However, their risk-benefit profile has been recently brought into question by 2 randomized controlled trials (RCTs), STOP-IgAN and TESTING $[5,6]$. Due to the autoimmune basis of IgAN, a number of immunosuppressive agents commonly used in other glomerular diseases have been tested, including cyclophosphamide, rituximab, azathioprine, and mycophenolate, but there has been no consistent evidence to support the routine use of any of these agents [7].

It is now widely accepted that IgAN occurs due to multiple "hits": an increase in circulating "pathogenic" poorly $\mathrm{O}$-galactosylated $\mathrm{IgA} 1$, production-specific IgG and IgA autoantibodies that recognize this form of IgA1, IgA1-immune complex formation, and their deposition in the glomerular mesangium, triggering renal damage [8]. In this review, we will summarize emerging information regarding the pathogenesis of IgAN and describe how this has informed the development of new therapeutic strategies with the aim of intervening at each of these stages.

\section{IgA1 O-Galactosylation}

In humans, $\operatorname{Ig} \mathrm{A}$ exists as 2 isoforms, $\operatorname{IgA} 1$ and $\operatorname{IgA}$, which in turn exist as monomers or polymers, and is found within mucosal and circulating compartments. IgA1 differs from IgA2 in that it contains an extended 18 amino acid hinge region, between the first and second constant domains of the $\alpha 1$ heavy chain (Fig. 1a). This is variably $\mathrm{O}$-galactosylated by the addition of $\mathrm{O}$-glycans chains containing terminal galactose to serine and threonine residues at specific positions. At mucosal surfaces,

Fig. 1. IgA1 galactosylation. The IgA1 molecule contains a hinge region between the $\mathrm{CH} 1$ and $\mathrm{CH} 2$ domains, which is variably glycosylated. Six of the 9 possible glycosylation residues (indicated in red) can be elongated by the stepwise addition of monosaccharides, in $O$-linkage. a The addition of $O$-glycans starts by the binding of GalNAc residues to IgA1-hinge region serine or threonine residues by the GalNAcT2 enzyme, forming the Tn antigen. This can then be elongated by the addition of galactose residues by C1GALT1 and its chaperone Cosmc to form the T antigen, or alternatively the addition of NeuAc by ST6GalNac-II (STn antigen). The galactose residues can be extended by the addition of NeuAc by ST3Gal (ST antigen). IgA1 molecules may therefore possess a complex variety of glycosylation patterns. b Poorly galactosylated
IgA1 is the most abundant immunoglobulin and plays an important role in the host defense against microbial invasion. The role of IgA within the circulation is unclear. IgA1, with its variably galactosylated hinge region, only exists in humans and higher primates, and therefore, drug development has been hindered by the lack of an accurate and easily reproducible rodent model.

Serum IgA is composed mainly of the monomeric form, but in IgAN, there is an increase in circulating IgA1 that is polymeric and lacks terminal galactose residues in its hinge region (termed "poorly galactosylated IgA1," Fig. 1b) [9]. This form of IgA1 is usually most abundant within the mucosal compartment. In the circulation however, poorly galactosylated IgA1 may form immune complexes, with itself or with IgA and IgG antibodies directed against the hinge region. Lectin binding experiments, later confirmed by mass spectrometry, demonstrated that the IgA isolated from the mesangial deposits in IgAN is predominantly poorly galactosylated IgA1 $[10,11]$. Serum levels of poorly galactosylated IgA1 have subsequently been demonstrated to correlate with renal prognosis [12].

Following mesangial deposition of IgA1, in susceptible individuals, a number of inflammatory and fibrotic processes are upregulated that lead to renal damage (Fig. 2). However, since raised serum levels of poorly galactosylated IgA1 may also be found in healthy individuals, notably in first degree relatives of patients with IgAN, and glomerular IgA 1 deposition may occur in people who display no other features of clinically overt disease, additional factors to mesangial IgA1 deposition are likely to play a key role in the progression of IgAN [13].

$O$-Galactosylation of the IgA1-hinge region is influenced by a number of genetic and epigenetic factors, some of which have recently been characterized. Of note, core 1 beta 1, 3-galactosyltransferase (C1GalT1) is the enzyme responsible for attaching galactose to the $\operatorname{IgA} 1$

IgA1 molecules, found at increased levels in IgAN, miss the addition of galactose residues to the hinge region by the lower activity of C1GalT1/Cosmc enzymes and/or the addition of NeuAc residues to GalNAc by ST6GalNAc-II. P, proline; S, serine; T, threonine; GalNAc, N-acetylgalactosamine; Gal, galactose; NeuAc, neuraminic acid; $\mathrm{CH} 1,2,3$, constant heavy chain $1,2,3$; $\mathrm{VH}$, variable heavy chain; CL, constant light chain; VL, variable light chain; GalNAcT2, polypeptide N-acetylgalactosaminyltransferase 2; C1GalT1, core 1 beta 1, 3-galactosyltransferase; Cosmc, core 1 33 GalT-specific molecular chaperone; ST6GalNac-II, ST6 Nacetylgalactosaminide alpha-2,6-sialyltransferase 2, ST3Gal, alpha2,3-sialyltransferase. 
hinge region. A common variant in the noncoding region of the C1GALT1 gene has been identified, which is associated with increased levels of poorly galactosylated IgA1 [14]. This association with the noncoding area of the gene would be consistent with changes in the regulation of C1GALT1 expression, possibly in specific microenvironments such as the mucosa, rather than a change in protein structure, which would affect all C1GALT1-expressing cell types. This finding is not restricted to those with IgAN but was also seen in healthy subjects and patients with membranous nephropathy, and in white and Chinese populations, implying that changes in IgA1 O-galactosylation are heritable and influenced by the C1GALT1 gene. Interestingly, although IgAN has a higher preva-

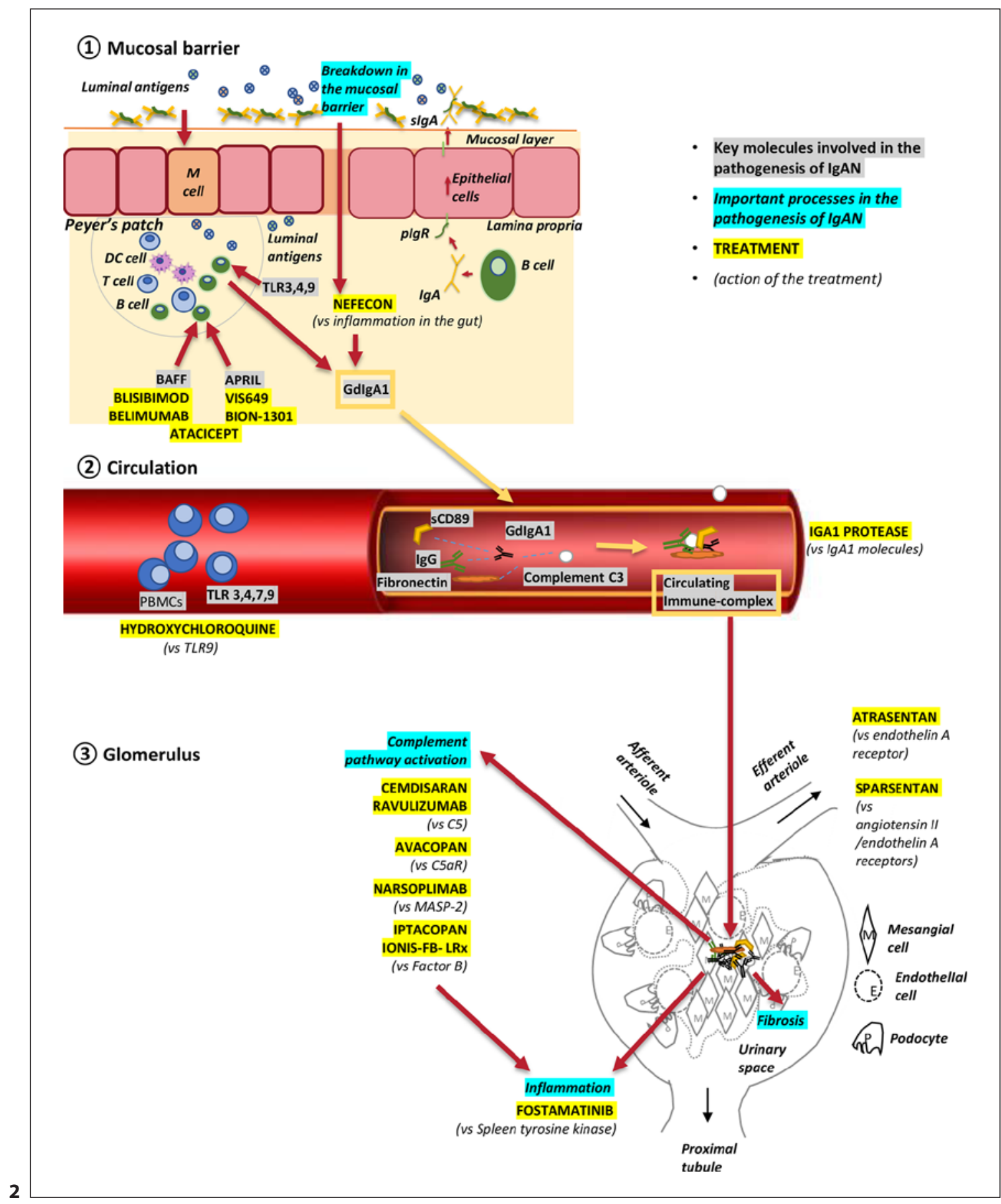

(For legend see next page.) 
lence in Chinese than in white populations, levels of poorly galactosylated IgA1 were observed to be lower in Chinese IgAN patients than in white IgAN patients, and this corresponded with a lower frequency of the C1GALT1 risk haplotype [15]. This raises important questions regarding whether other pathogenetic mechanisms act at differing levels in different populations and the relative importance of these mechanisms.

MicroRNAs (miRNAs) are short noncoding RNA molecules that modulate gene expression. Expression of the miRNA, miR-148b, has been shown to be increased in peripheral blood mononuclear cells (PBMCs) from patients with IgAN, and in vitro upregulation of miR-148b in PBMCs resulted in reduced expression of $\mathrm{C1GalT1} 1$ and increased secretion of poorly galactosylated IgA1 [16]. A binding site for miR-148b within the C1GALT1 gene has been identified [16].

A key area of interest in IgAN is therefore targeting these finely controlled processes (a further discussion of these can be found elsewhere [17]). Advances in technologies, for example, the ability to manipulate genes or miRNAs involved in IgA1 O-galactosylation, will likely be harnessed in the near future to design new therapies.

\section{The Origin of Poorly O-Galactosylated IgA1 in IgAN}

There has been long-standing interest in the links between IgAN and the mucosal immune system, with the observation that patients may experience episodes of visible hematuria that coincide with a respiratory or gastrointestinal infection. IgA, and in particular poorly galactosylated IgA1, is produced primarily at respiratory and gut mucosal surfaces, within the mucosa-associated lymphoid tissue (MALT). Important MALT sites implicated in the pathogenesis of IgAN are the tonsils and the gut

Fig. 2. A proposed pathogenesis for IgAN and potential therapeutic targets. 1. Mucosal barrier: Luminal antigens passing through the $\mathrm{M}$ cells of the epithelial barrier of the gut are recognized by TLR3, TLR4, and/or TLR9 on B cells in the Peyer's patches, leading to the release of poorly galactosylated IgA1 (also known as galactose-deficient IgA1 or GdIgA1). IgA produced by B cells in the mucosal barrier is bound by the pIgR. This process is crucial for the transcytosis of IgA molecules through the epithelial barrier and the release into the mucosal layer. Here, IgA molecules are present as sIgA, carrying the cleaved part of their pIgR, the secretory component. The production of IgA is sustained by the increase in BAFF and APRIL, key promoters of the maturation of B cells. In IgAN, a breakdown in the integrity of the intestinal mucosal layer may be responsible for the introduction of GdIgA1 from the mu- (gut-associated lymphoid tissue [GALT]). The GALT produces the most IgA of all the MALT sites, and this is concentrated within specialized collections of lymphoid follicles called the Peyer's patches, in the distal ileum.

Growing evidence supports an important link between the gut mucosal system and IgAN. The incidence of certain gastrointestinal disorders, including inflammatory bowel disease and coeliac disease, is higher in patients with IgAN than in the general population [18]. Consistent with this, genome-wide association studies (GWAS) have identified multiple risk alleles for IgAN that are also associated with the immune response against intestinal pathogens, IgA synthesis within the gut, integrity of the intestinal epithelial barrier, and inflammatory bowel disease [19]. Two separate mouse models, a B-cell activating factor (BAFF)-overexpressing transgenic mouse and a humanized transgenic mouse that expresses human IgA1 and CD89, have been shown to be dependent on the presence of gut commensal flora to display an IgAN-renal phenotype $[20,21]$. Intriguingly, a cross-sectional study demonstrated that patients with progressive IgAN may display differences in their gut microbiota profile [22]. Gut sensitivity to various mucosal antigens has also been reported in IgAN, although there is no clear evidence that any specific dietary modification has a clinically beneficial effect $[23,24]$.

Therapies directed towards the GALT have therefore become a key area of interest in IgAN. A targeted release formulation of budesonide (Nefecon) has been developed that is designed to specifically release the active drug at the distal ileum, targeting the Peyer's patches (Fig. 2). The NEFIGAN study was a Phase II RCT, studying the effects of Nefecon in IgAN (Table 1) [25]. A significant reduction in proteinuria and stabilization of kidney function was observed in those treated with $16 \mathrm{mg}$ Nefecon daily for 9 months, compared to the placebo group where the

cosal layer. 2. Circulation: Raised levels of GdIgA1 are found in the serum in IgAN. GdIgA1 may originate from the gut mucosal barrier and/or from circulating PBMCs. The activation of TLRs on PBMCs could lead to increased production of GdIgA1. Circulating GdIgA1 forms complexes with other molecules, including IgG, sCD89, fibronectin, and complement C3. 3. Glomerulus: The IgA1-containing immune complexes deposit in the glomeruli, initiating complement pathway activation, inflammatory and fibrotic processes, and renal damage. Processes involved are highlighted in blue. Key molecules involved are highlighted in gray. New therapies in development are highlighted in yellow. PBMC, peripheral blood mononuclear cells; pIgR, polymeric immunoglobulin receptor; $\mathrm{M}$, microfold; sCD89, soluble CD89. 
Table 1. Ongoing or recently completed clinical trials of novel therapies in IgAN

\begin{tabular}{|c|c|c|c|}
\hline \multicolumn{4}{|l|}{ Gut directed therapy } \\
\hline TRF-budesonide (nefecon) & $\begin{array}{l}\text { Phase III RCT (NeflgArd) - ongoing } \\
\text { NCT03643965 }\end{array}$ & $\begin{array}{l}\text { Corticosteroid targeting B cells in } \\
\text { GALT }\end{array}$ & \\
\hline \multicolumn{4}{|l|}{ BAFF/APRIL directed therapies } \\
\hline Atacicept & $\begin{array}{l}\text { Phase II RCT - terminated early (slow } \\
\text { recruitment) [29] } \\
\text { NCT02808429 } \\
\text { Phase II RCT (ORIGIN) - ongoing } \\
\text { NCT04716231 }\end{array}$ & Blocks BAFF and APRIL signaling & $\begin{array}{l}\text { Reduction in GdlgA1 } \\
\text { Reduction in proteinuria }\end{array}$ \\
\hline Blisibimod & $\begin{array}{l}\text { Phase II RCT (BRIGHT-SC) - completed } \\
\text { NCT02062684 }\end{array}$ & Selective BAFF inhibitor & Results awaited \\
\hline $\mathrm{BION}-1301$ & $\begin{array}{l}\text { Open-label Phase II trial - ongoing } \\
\text { NCT03945318 }\end{array}$ & Monoclonal Ab against APRIL & \\
\hline Bortezomib & $\begin{array}{l}\text { Phase II open-label pilot study - completed } \\
\text { [31] }\end{array}$ & $\begin{array}{l}\text { Proteasome inhibitor - depletes } \\
\text { plasma cells }\end{array}$ & Reduction in proteinuria \\
\hline \multicolumn{4}{|l|}{ TLR directed therapy } \\
\hline Hydroxychloroquine & Phase II RCT - completed [37] & Inhibits TLR signaling & Reduction in proteinuria \\
\hline \multicolumn{4}{|c|}{ Immunoreceptor signaling directed therapy } \\
\hline Fostamatinib & Phase II RCT (SIGN) - completed [53] & Syk inhibitor & $\begin{array}{l}\text { Nonsignificant reduction in } \\
\text { proteinuria - full results awaited }\end{array}$ \\
\hline \multicolumn{4}{|l|}{ Complement pathway inhibitors } \\
\hline Iptacopan (LNP023) & $\begin{array}{l}\text { Phase III RCT (APPLAUSE-IgAN) - ongoing } \\
\text { NCT04578834 }\end{array}$ & $\begin{array}{l}\text { Factor B inhibitor - inhibits } \\
\text { alternative pathway }\end{array}$ & \\
\hline IONIS-FB-LRx & $\begin{array}{l}\text { Open-label Phase II trial - ongoing } \\
\text { NCT04014335 }\end{array}$ & $\begin{array}{l}\text { Factor B inhibitor - inhibits } \\
\text { alternative pathway }\end{array}$ & \\
\hline Sparsentan & $\begin{array}{l}\text { Exploratory Phase Il open-label mechanistic } \\
\text { study (SPARTAN) - ongoing } \\
\text { NCT04663204 } \\
\text { Phase III RCT (PROTECT) - ongoing } \\
\text { NCT03762850 }\end{array}$ & Combined ARB and ETA-R blocker & \\
\hline Atrasentan & $\begin{array}{l}\text { Phase III RCT (ALIGN) - ongoing } \\
\text { NCT0 } 4573478\end{array}$ & ETA-R blocker & \\
\hline
\end{tabular}

Data from www.clinicaltrials.gov (accessed on 13 July 2021). TRF, targeted release formulation; RCT, randomized controlled trial; BAFF, B-cell activating factor; APRIL, a proliferation-inducing ligand; GdlgA1, galactose-deficient IgA1; Ab, antibody; MASP-2, mannose-binding protein-associated serine protease 2; ARB, angiotensin receptor blocker; GALT, gut-associated lymphoid tissue; TLR, toll-like receptor; Syk, spleen tyrosine kinase; ETA-R, endothelin-A receptor; eGFR, estimated glomerular filtration rate.

estimated glomerular filtration rate (eGFR) fell by an average of $4.7 \mathrm{~mL} / \mathrm{min} / 1.73 \mathrm{~m}^{2}$. The Phase III NeflgArd trial, comparing treatment with Nefecon or placebo for 9 months in addition to maximum tolerated RAAS blockade, has recently completed recruitment and will examine efficacy over a 2-year follow-up period (ClinicalTrials. 


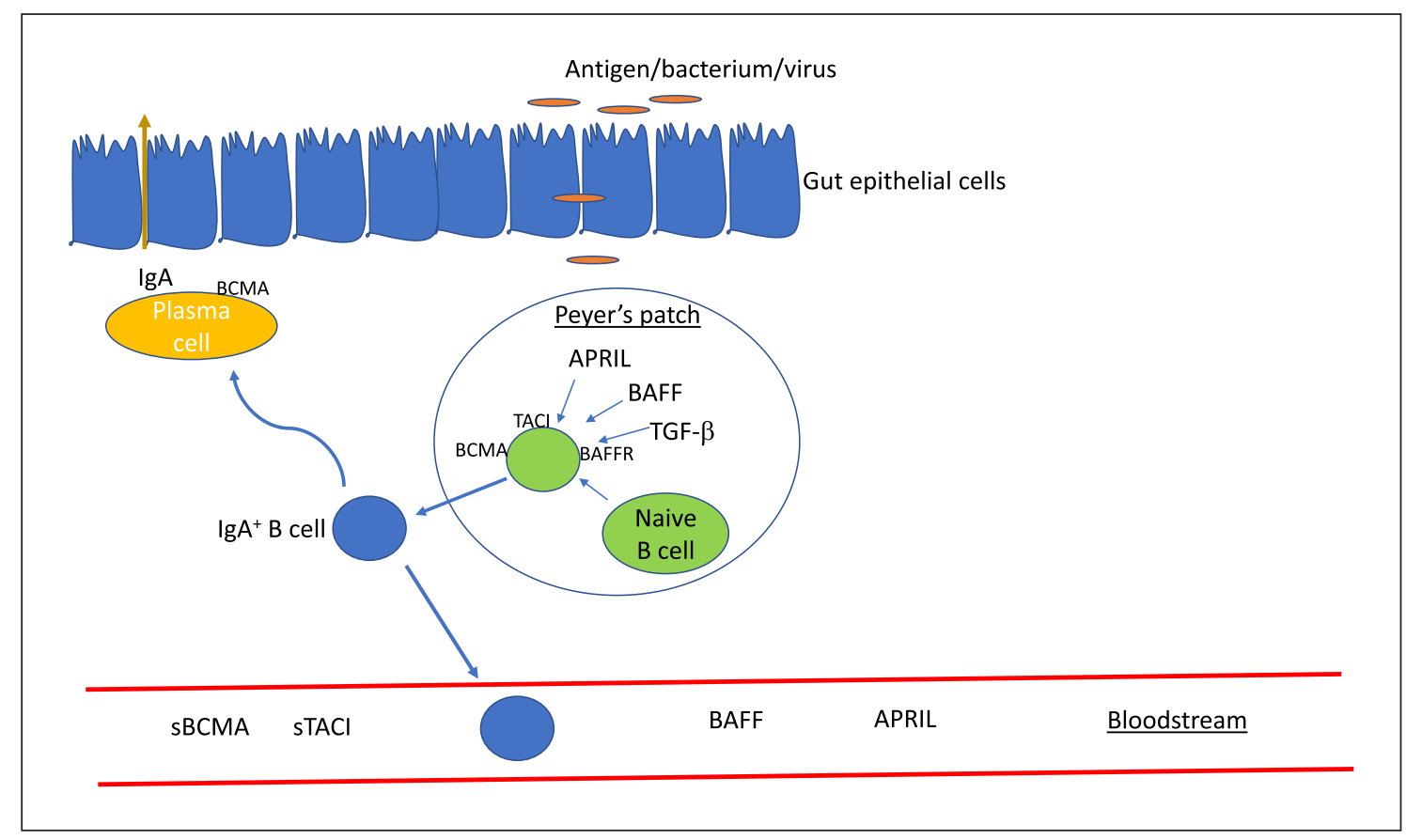

Fig. 3. Control of mucosal B-cell homeostasis by BAFF and APRIL. BAFF and APRIL are essential for the maturation and survival of GALT B cells, and IgA class switch recombination. BAFF and APRIL bind to the B-cell surface receptors TACI and BCMA at different stages in B-cell maturation, and BAFF also binds to

gov identifier: NCT03643965). Investigating in detail the effects of targeting the GALT in this way may shed additional light on the importance of regulation of mucosal IgA1 production in IgAN. Potential differences in the composition of the gut microbiome in patients with IgAN compared to healthy subjects are intriguing and require further study with larger numbers of subjects, followed longitudinally, to assess whether this is a consistent finding, and if manipulation of the gut microbiome, for example, with probiotics, may be a future avenue for therapeutic intervention [26].

\section{Modulation of Mucosal IgA Production in IgAN: The Role of BAFF and APRIL}

Control of B-cell homeostasis in the Peyer's patches is a tightly regulated process controlled by BAFF, also called $B$ lymphocyte activator, a proliferation-inducing ligand (APRIL), and their receptors, BAFF receptor (BAFF-R), $\mathrm{B}$-cell maturation antigen, and transmembrane activator and calcium modulator and cyclophilin-ligand interactor

Pathogenesis and Treatment of IgAN
BAFF-R. BAFF, B-cell activating factor; APRIL, a proliferatinginducing ligand; TACI, transmembrane activator and calcium modulator and cyclophilin-ligand interactor; BCMA, B-cell maturation antigen; BAFF-R, BAFF receptor; GALT, gut-associated lymphoid tissue.

(TACI) (Fig. 3) [17]. Raised levels of circulating BAFF and APRIL and an association between these cytokines and levels of circulating poorly galactosylated IgA1 suggest an important role for BAFF and APRIL in IgAN [17]. However, the data are not entirely consistent; it remains unclear whether BAFF or APRIL is the more relevant cytokine, or which is the dominant receptor subtype. The importance of the BAFF/APRIL system is supported by pre-clinical data in a BAFF-overexpressing transgenic mouse that displays a hyper-IgA syndrome and an IgANlike renal phenotype, and the efficacy of an anti-APRIL antibody in the ddY mouse model of IgAN, in terms of reducing IgA deposition and proteinuria [20, 27, 28]. In addition, a GWAS has identified TNFSF13 (which encodes APRIL) as a susceptibility locus for IgAN, and this risk variant is associated with higher levels of serum IgA in patients with IgAN [19].

Treatments inhibiting BAFF and APRIL signaling are being evaluated in clinical trials in IgAN. Atacicept is a fully humanized fusion protein containing the extracellular portion of TACI (a receptor for both BAFF and APRIL) that inhibits both signaling pathways. A 24-week 
interim analysis of data from a Phase II RCT showed a dose-dependent reduction of circulating poorly $\mathrm{O}$-galactosylated IgA1 and proteinuria in those treated with atacicept [29]. A Phase II trial of blisibimod, a selective inhibitor of BAFF, has been completed, and the full results are awaited (NCT02062684). Further clinical trials examining other BAFF and APRIL inhibitors are ongoing, including BION-1301, a humanized IgG4 monoclonal antibody that inhibits APRIL, and VIS649, a humanized IgG2 monoclonal antibody, also directed against APRIL (NCT03945318 and NCT04287985, respectively). IgG levels are known to fall after BAFF and/or APRIL inhibition. Whether these therapies have a specific effect on anti-IgA1 IgG autoantibodies is not yet clear but is an important area of interest.

In contrast to targeting BAFF and APRIL, depletion of CD19/20+ B cells with rituximab has not been shown to be effective in a small open-label trial in IgAN [30]. Over a 1-year period, patients treated with rituximab had no improvement in proteinuria or kidney function compared to baseline or the control group, experienced more adverse events, and importantly had no reduction in serum levels of poorly galactosylated IgA1 or anti-IgA1 IgG autoantibodies, despite effective circulating B-cell depletion. This has led to the hypothesis that plasmablasts, plasma cells, or other tissue-resident B cells may play an important role in the production of poorly galactosylated IgA1 in IgAN. Therapies targeting other B-cell populations are currently being explored, and a pilot study of the proteasome inhibitor bortezomib recently demonstrated a reduction of proteinuria in 8 patients with IgAN [31].

\section{Toll-Like Receptors}

Control of BAFF and APRIL in the gut is, in part, modulated through Toll-like receptor (TLR) activation. TLRs mediate the host immune response to microbial stimuli, by recognition of pathogen-associated molecular patterns and danger-associated molecular patterns. TLRs are predominantly found on the surface of immune cells, but are also seen on other cell types including mucosal epithelial cells, where they face continuous microbiological stimuli. Activation of TLRs leads to the activation of a number of signaling cascades, resulting in production of interferons and release of pro-inflammatory cytokines that direct the adaptive immune response.

Links between a variety of TLRs, BAFF, and APRIL signaling and IgAN have been reported. Exposure of IgAN-prone ddY mice to the TLR9 ligand CpG-oligodeoxynucleotide increased the production of poorly galactosylated IgA, and exacerbated kidney injury, in a manner that was dependent on APRIL and IL-6 [32]. Stimulation of TLR9 also induced increased expression of APRIL by $\mathrm{B}$ cells isolated from tonsillar germinal centers in patients with IgAN, and clinically, increased APRIL expression by tonsillar germinal centers was linked with severity of proteinuria [33]. Similar to TLR9, TLR3 is activated by viral stimuli and may play a role in the pathogenesis of IgAN. In both patients with IgAN and in a rat model of IgAN, expression of TLR3 and BAFF was increased in blood and tonsillar samples [34]. Recently, increased expression of TLR7 was also shown in PBMCs from patients with IgAN, and this was associated with higher serum levels of poorly galactosylated IgA 1 and markers of renal inflammation [35]. Renal expression of TLR4, TLR7, TLR8, and TLR9 has also been shown to be increased in IgAN, and associated with lower eGFR and increased proteinuria [36].

Hydroxychloroquine inhibits TLR9 and to a lesser extent TLR7 and TLR8 signaling. Given the proposed role of TLRs in IgAN, a RCT has been performed which demonstrated reduction of proteinuria in patients treated with hydroxychloroquine for 6 months [37]. However, this RCT was conducted in a small number of patients and was limited to Chinese patients, and therefore, the findings may not be generalizable. Hydroxychloroquine may be a potential therapeutic option and is known to be well tolerated and is of low cost, and therefore may have a large potential impact. Larger studies are required before any recommendation is possible, and in addition, a number of specific TLR antagonists are currently in development [38].

\section{Circulating IgA1-Immune Complexes in IgAN}

Elevated levels of circulating immune complexes containing poorly galactosylated IgA 1 are commonly detected in patients with IgAN. In vitro, both polymeric IgA1 and IgG-IgA1-immune complexes, but not monomeric IgA1, have been shown to stimulate human mesangial cells, suggesting that components of the IgA1-immune complex play an important role in the pathogenesis of IgAN [39].

A key area of interest is the role of IgA and IgG antibodies with specificity for the poorly galactosylated IgA1 hinge region. IgG is variably found to be co-deposited with IgA in the mesangium in IgAN and when present has been linked to a worse prognosis [40]. In the serum, anti-IgA 1 hinge region IgG antibodies were included in a panel of biomarkers reported to discriminate IgAN from other glomerular diseases [41]. Serum levels of both IgA1 
and IgG anti-IgA1 hinge region antibodies have been associated with progression of IgAN [42]. IgG anti-IgA1 hinge region antibodies have also been detected within mesangial deposits in IgAN [43]. The ability of IgG to bind to poorly galactosylated IgA1 has been shown to be dependent on a somatic mutation causing an alanine to serine substitution in the CD3 domain of the variable region of the gene encoding for IgG [44].

One serum protein of interest that has been identified within IgA1-immune complexes in IgAN is the myeloid IgA receptor, CD89, which exists in both membranebound and soluble forms. There are conflicting reports regarding the clinical significance of IgA1-CD89 complexes in IgAN, and in particular, mesangial deposition of CD89 in IgAN has not been consistently demonstrated [45]. Glomerular deposition of Streptococcal M proteins in patients with IgAN and IgA vasculitis has been reported, again suggesting a link between mucosal infections and IgAN [46].A greater understanding of the constituents of circulating and mesangial IgAl-immune complexes may aid our understanding of the heterogeneity of clinical presentations in IgAN and potentially offer novel targets for therapeutic intervention.

\section{Mesangial Deposition of IgA1 and Glomerular Injury}

A number of in vitro studies have aimed to define the mechanisms by which deposition of IgA1-immune complexes leads to mesangial cell activation, proliferation, and release of pro-inflammatory and profibrotic mediators. Polymeric IgA1, poorly galactosylated IgA1, secretory IgA, and IgG-IgA1-immune complexes have all been reported to increase synthesis of pro-inflammatory cytokines by human mesangial cells [17]. The mechanisms by which mesangial cells recognize and respond to IgA1 are not completely understood. Binding of IgA-containing immune complexes to mesangial cell receptors is a possible critical pathogenic step and hence a potential therapeutic target. Therefore, intensive research efforts have been directed towards identifying candidate mesangial IgA1 receptors. Out of the known IgA receptors, only the transferrin receptor and $\mathrm{Fc} \alpha / \mu \mathrm{R}$ were previously demonstrated to be expressed by mesangial cells. More recently, a novel IgA receptor, $\beta$-1,4-galactosyltransferase 1 , has been characterized and its expression by mesangial cells was increased in patients with IgAN [47].

One treatment strategy that is being explored is the inhibition of downstream inflammatory pathways that may be activated following mesangial IgA1 deposition.
Spleen tyrosine kinase (Syk) is a key protein involved in immunoreceptor signaling and activation of downstream pathways, including c-Jun and p38 MAPK [48]. Syk is expressed in different cell types, including myeloid and renal cells, and responds to the activation of B-cell receptors, $\mathrm{Fc}$ receptors, and IgA binding to beta 1,4 galactosyltransferase on the surface of human mesangial cells [47, 49]. Glomerular expression of Syk has been shown to be upregulated in IgAN and correlated with serum creatinine levels at the time of biopsy [50]. Inhibition of Syk signaling in vitro, either by siRNA knockdown or use of an active metabolite of the inhibitor fostamatinib, has been shown to block the inflammatory response of human mesangial cells following exposure to IgA1 from IgAN patients [51].

Syk inhibition is therefore a potentially attractive therapeutic strategy, especially as the Syk inhibitor fostamatinib has been shown to be well tolerated in large RCTs in chronic immune thrombocytopenia, and has been licensed for use in this condition [52]. A Phase II RCT of Syk inhibition in IgAN (SIGN: Syk inhibition in IgAN) has recently been completed. Preliminary results indicated a dose-dependent reduction in proteinuria in those treated with fostamatinib with a baseline urine PCR $>1,000 \mathrm{mg} / \mathrm{g}$, although this did not reach statistical significance [53]. The full results from this trial are awaited.

\section{Complement Pathway Activation and IgAN}

The complement system is a critically controlled trio of pathways composed of multiple heat-labile proteins, which provide an important link between the innate and adaptive immune systems. The 3 pathways, classical, alternative, and lectin, are differentially triggered but converge at the point of activating complement component 3 (C3) by the formation of pathway-specific C3 convertases. C3 activation ultimately results in the production of anaphylatoxins (C3a and C5a), leading to the recruitment of inflammatory mediators, and formation of the membrane attack complex, resulting in cell lysis (Fig. 4). Complement activation has been recognized to play an important role in several glomerular diseases, including atypical hemolytic uremic syndrome, C3 glomerulopathy, dense deposit disease, lupus nephritis, ANCA-associated vasculitis, and membranous nephropathy $[54,55]$.

C3 is co-deposited with mesangial IgA1 in the majority of patients with IgAN, and the near ubiquitous absence of C1q implies that in IgAN, alternative and/or lectin, but not classical, pathways are activated. Detection of 


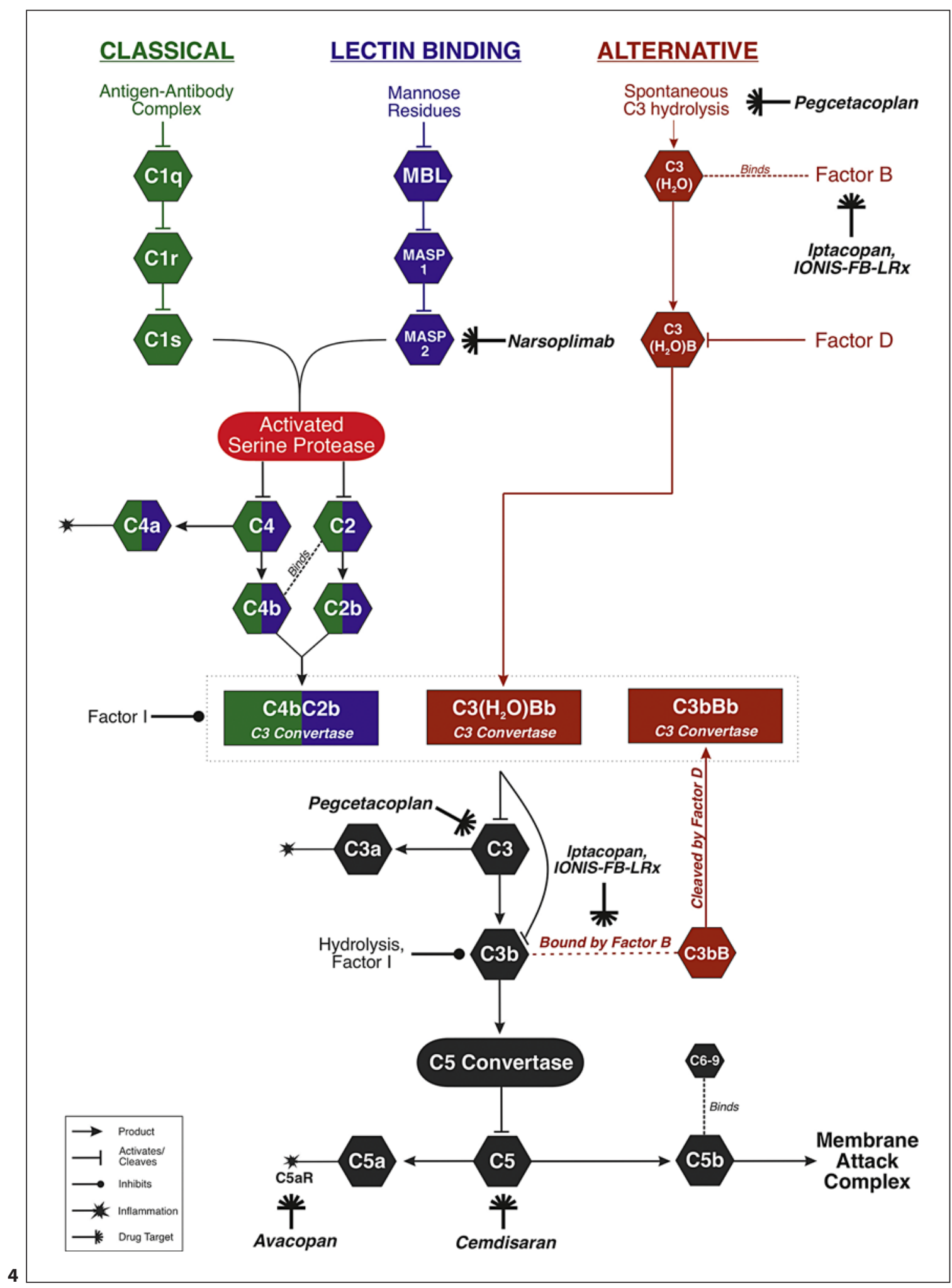

(For legend see next page.) 
alternative pathway components in renal biopsies, serum, and urine from IgAN patients has all been associated with worse renal outcome [56]. Lectin pathway activation can be demonstrated in a subgroup of patients with IgAN. In 2 independent IgAN cohorts, glomerular deposition of lectin pathway components was detected in approximately $25 \%$ of cases and was associated with worse disease severity $[57,58]$.

The origin of mesangial C3 deposits in IgAN has not been established, and potential sources include the circulation, either as a component of circulating IgA immune complexes or in situ fixation triggered following IgA immune complex deposition, and/or local production by resident mesangial cells or infiltrating immune cells [56]. Circulating C3 activation fragments can be detected in around half of patients with IgAN [56]. The ratio of serum IgA to $\mathrm{C} 3(\mathrm{Ig} A / \mathrm{C} 3)$ has been reported as a biomarker for diagnosis and progression of IgAN [59]. More recently, the ratio of poorly galactosylated IgA1 to C 3 was reported to be predictive of progressive renal decline in IgAN, and this was independent of clinical and histological characteristics [60]. These reports are confined to cohorts from Asia, and further studies are needed to see whether these changes are also seen in other patient cohorts.

Two separate GWAS reported an association between the CFH/CFHR locus and IgAN. Deletion of complement factor-H-related protein-1 and protein-3 was shown to be protective against the risk of developing IgAN. Complement factor-H-related protein- 1 and complement factor$\mathrm{H}$-related protein-1 compete with the binding of factor $\mathrm{H}$ to $\mathrm{C} 3$, and their deletion results in uninhibited factor $\mathrm{H}-\mathrm{C} 3$ binding, and downregulation of the alternative pathway.

Fig. 4. The complement system and therapeutics. The complement system can be activated by 3 arms. The classical pathway (green) is activated by antigen-antibody complexes, which bind $\mathrm{C} 1 \mathrm{q}$ inducing a conformational change to permits the associated $\mathrm{C} 1 \mathrm{r}$ to cleave $\mathrm{C} 1 \mathrm{~s}$ into an activated serine protease. $\mathrm{C} 1$ s subsequently cleaves $\mathrm{C} 4$ to produce $\mathrm{C} 4 \mathrm{a}$, a strong inflammatory mediator, and $\mathrm{C} 4 \mathrm{~b}$. C4b binds $\mathrm{C} 2$ permitting it to be cleaved by the serine protease, producing $\mathrm{C} 2 \mathrm{~b}$. $\mathrm{C} 4 \mathrm{~b}$ and $\mathrm{C} 2 \mathrm{~b}$ bind to produce a $\mathrm{C} 3$ convertase. The lectin binding pathway (purple) is activated by mannose moieties commonly found on microbial surfaces, but also on poorly $O$-galactosylated IgA1. These moieties are bound by a MBL, which activates MASP-1 and MASP-2, which are analogous to C1r and $\mathrm{C} 1 \mathrm{~s}$ of the classical pathway. MASP-2 activation leads to C4bC2b production as detailed above. MASP-2 can be therapeutically inhibited by narsoplimab. The alternative pathway (red) is a constantly activated pathway, triggered by the hydrolysis of C3 (inhibited by Pegcetacoplan) thioester bonds. $\mathrm{C} 3\left(\mathrm{H}_{2} \mathrm{O}\right)$ is bound by Fac-
The increasing number of targeted complement pathway inhibitors has led to a great deal of interest in complement-directed therapies in IgAN. Initial case reports of C5 inhibition using eculizumab reported temporary slowing of renal decline in IgAN with rapidly progressive disease providing proof of concept for further evaluation of complement inhibition in IgAN [56]. A number of ongoing trials are evaluating drugs that target the alternative, lectin, and terminal complement pathways. Iptacopan (LNP023) is an oral selective factor B inhibitor that targets alternative pathway activation. A Phase III RCT is currently being conducted (NCT04578834), and this agent is also being studied in other conditions including membranous nephropathy, C3 glomerulopathy, atypical HUS, and paroxysmal nocturnal hemoglobinuria. IONIS-FB-LRx is an antisense inhibitor directed against Factor B mRNA, and its safety and efficacy are also being tested in patients with IgAN in a small single-arm openlabel study (NCT04014335). Cemdisiran is an RNA interfering therapeutic agent that blocks hepatic production of C5, and a Phase II RCT is underway to investigate its effects in IgAN (NCT03841448). C5 is also being targeted in a Phase II study of the long-acting C5 inhibitor ravulizumab (NCT04564339). The C5a receptor can be specifically targeted by the oral small molecule inhibitor Avacopan (CCX168), which has been shown to be safe and effective as part of treatment for ANCA-associated vasculitis in a Phase III trial [61]. Results from an openlabel Phase II trial in IgAN have been presented, which demonstrated a reduction in proteinuria by 12 weeks, in 6 out of 7 patients tested [62]. Narsoplimab (OMS-721) is a human monoclonal antibody that targets mannosebinding protein-associated serine protease 2 (MASP-2), a

tor $\mathrm{B}$, which renders the complex susceptible to cleavage by Factor D. This produces the $\mathrm{C} 3$ convertase $\mathrm{C} 3\left(\mathrm{H}_{2} \mathrm{O}\right) \mathrm{Bb}$. Factor $\mathrm{B}$ can be therapeutically inhibited by Iptacopan or IONIS-FB-LRx. The common pathway (black) is activated by any of the $\mathrm{C} 3$ convertases, which cleave C3 (inhibited by Pegcetacoplan) to C3a, an inflammatory mediator, and $\mathrm{C} 3 \mathrm{~b}$. C $3 \mathrm{~b}$ is further acted upon by the $\mathrm{C} 3$ convertases to produce a C5 convertase, which cleaves C5 (can be inhibited by Cemdisiran) to produce $\mathrm{C} 5 \mathrm{a}$, a potent inflammatory mediator (its receptor can be inhibited by Avacopan) and C5b. $\mathrm{C} 5 \mathrm{~b}$ is serially bound by $\mathrm{C} 5, \mathrm{C} 6, \mathrm{C} 7, \mathrm{C} 8$, and $\mathrm{C} 9$ to form the MAC, which is capable of cell lysis. C3b can also be bound by Factor B of the alternative pathway to form $\mathrm{C} 3 \mathrm{bB}$, which can be cleaved by Factor $\mathrm{D}$ to produce the $\mathrm{C} 3$ convertase $\mathrm{C} 3 \mathrm{bBb}$. $\mathrm{C} 3 \mathrm{~b}$ activity is regulated by hydrolysis and Factor I, which inhibit its activity. MASP2, mannose-binding protein-associated serine protease 2; MAC, membrane attack complex; MBL, mannose-binding lectin. 
critical effector enzyme of the lectin pathway. A case report demonstrated stabilization of renal function in IgA vasculitis [63], and a staged Phase II study demonstrated that narsoplimab treatment was well-tolerated and reduced proteinuria in high-risk patients with IgAN, who had proteinuria $>1 \mathrm{~g} /$ day [64]. A Phase III RCT of narsoplimab in IgAN is currently open to recruitment (NCT03608033).

\section{Nonimmune Modulators}

Sparsentan is a first-in-class combined angiotensin receptor blocker (ARB) and endothelin-A receptor (ETA$\mathrm{R}$ ) antagonist, which has both ARB and podocyte-protective effects as well as a number of other putative anti-fibrogenic, anti-inflammatory, and antioxidant actions and has shown promising results in terms of proteinuria reduction in a Phase II study in FSGS [65]. Sparsentan is currently being tested in IgAN in the Phase III PROTECT RCT and in a separate exploratory mechanistic sub-study (SPARTAN) (NCT03762850 and NCT04663204). A separate Phase III trial of the ETA-R antagonist, atrasentan, is also being conducted (ALIGN) (NCT04573478).

The sodium-glucose cotransporter 2 inhibitors (SGLT2i) are a novel class of medications that have recently been introduced for the treatment of diabetes mellitus. These work by enhancing glycosuria by blocking the proximal tubular SGLT2 cotransporter and inhibiting the entry of glucose into the proximal tubular cells. It has become apparent that these medications also provide significant renal and cardiovascular benefits that are independent of their anti-glycemic effects. The DAPA-CKD trial randomized 4,304 participants with eGFR between 25 and $75 \mathrm{~mL} / \mathrm{min} / 1.73 \mathrm{~m}^{2}$, with or without type 2 diabetes mellitus, and was stopped early due to efficacy. Over a median of 2.4 years, there was a significant reduction in those reaching a primary outcome event of a sustained decrease in eGFR of at least $50 \%$, end-stage kidney disease, or death from a renal or cardiovascular cause in those taking dapagliflozin rather than placebo [66]. A prespecified analysis of 270 participants with IgAN included in this trial again demonstrated a reduction in the primary outcome in those taking dapagliflozin, and also reductions in eGFR decline and albuminuria [67]. It should be noted that around $14 \%$ of these patients had coexistent diabetes mellitus and that not all patients had a biopsy-proven diagnosis of IgAN. Importantly, there was no run-in period in this trial where RAAS blockade was maximized, and therefore, it is possible that some pa- tients could have responded to this measure alone, and ideally, a dedicated trial in IgAN incorporating this would allay these concerns [68]. The EMPA-KIDNEY trial, currently underway, will provide additional data regarding the additional effect of sodium-glucose cotransporter 2 inhibitors in CKD compared to conventional therapies [69].

\section{Future Areas of Interest}

To date, no treatment directly targets the circulating IgA1-immune complexes that are seen in IgAN. One potential area of interest is the IgA1-specific proteases. These are produced by several bacterial species, including $S$. pneumoniae, $N$. meningitidis, $N$. gonorrhoeae, and $H$. influenzae, and specifically cleave the IgA1-hinge region, so do not affect IgA2. The use of an IgA1 protease was tested in the a1KI-CD89 Tg mouse model of IgAN and resulted in reduced mesangial IgA deposits and hematuria [70]. In studies with $\mathrm{BALB} / \mathrm{c}$ mice and in vitro tests, a consistent reduction of IgA1-IgG immune complexes has been observed [71]. The long-term safety and efficacy of this approach in humans have not yet been established. However, a similar approach is being tested with the use of the endopeptidase IdeS (immunoglobulin G degrading enzyme of Streptococcus pyogenes; Imlifidase), which rapidly cleaves all human IgG subclasses. Successful pilot studies have been carried out in highly sensitized patients undergoing renal transplantation to reduce donor-specific antibodies, and in anti-GBM disease, providing proof of concept that this treatment strategy may be effective in the treatment of autoimmune kidney disease $[72,73]$.

Despite the increased interest in performing clinical trials in IgAN, there remain important unmet needs. These include that the vast majority of clinical trials have not included patients with an $\mathrm{eGFR}<30 \mathrm{~mL} / \mathrm{min} / 1.73 \mathrm{~m}^{2}$. Recurrence of IgAN after renal transplantation has also not been widely studied. Finally, patients with IgA vasculitis have largely been excluded from clinical trials, despite many aspects of its pathogenesis being similar to IgAN.

\section{Conclusion}

Important advances have been made in the last decade in our understanding of the molecular mechanisms involved in the pathogenesis of IgAN. Deeper knowledge of the pathways involved has led to a resurgence of interest
Glomerular Dis 2022;2:15-29 DOI: $10.1159 / 000519973$
Scionti/Molyneux/Selvaskandan/Barratt/ Cheung 
in developing new treatment strategies in $\operatorname{IgAN}$, and in the number of Phase II and Phase III clinical trials evaluating new therapies. It is hopeful that within the next few years we will have the first approved treatment for IgAN and that this will be the first of many.

Many questions, however, continue to be unanswered, for example, the precise mechanism by which IgA deposits and leads to a response from mesangial cells, why IgAN causes progressive disease in certain patients and not others and the basis for the reported variations in disease severity and natural history between geographical areas. With the heterogeneity in presentation and outcomes, it has been suggested that IgAN may not represent a single disease and that mesangial IgA deposition is the final common endpoint for a range of disparate immunologically driven pathological pathways. Dissecting these different pathways will hopefully lead to the delivery of novel diagnostic and prognostic biomarkers, and the ability to individualize treatment to specific dysregulated pathways in patients with IgAN.

\section{Conflict of Interest Statement}

C.K.C. has received research grants from GlaxoSmithKline and Travere Therapeutics, and personal fees from Travere Therapeutics. J.B. has received research grants from Argenx, Calliditas Therapeutics, Chinook Therapeutics, Galapagos NV, GlaxoSmithKline, Novartis and Travere Therapeutics, and is medical/scientific advisor to Alnylam Pharmaceuticals, Argenx, Astellas Pharma, BioCryst Pharmaceuticals, Calliditas Therapeutics, Chinook Therapeutics, Dimerix, Galapagos NV, Novartis, Omeros, Travere Therapeutics, UCB, Vera Therapeutics, and Visterra.

\section{Funding Sources}

No research funding relevant to this article.

\section{Author Contributions}

K.S., K.M., J.B., and C.K.C. all contributed to reviewing the literature, manuscript writing, and review.

\section{References}

1 McGrogan A, Franssen CFM, de Vries CS. The incidence of primary glomerulonephritis worldwide: a systematic review of the literature. Nephrol Dial Transplant. 2011;26:41430.

2 Bumgardner GL, Amend WC, Ascher NL, Vincenti FG. Single-center long-term results of renal transplantation for IgA nephropathy. Transplantation. 1998;65:1053-60.

3 Silva FG, Chander P, Pirani CL, Hardy MA. Disappearance of glomerular mesangial IgA deposits after renal allograft transplantation. Transplantation. 1982;33:241-6.

4 Chapter 10: immunoglobulin a nephropathy. Kidney Int Suppl. 2012;2:209-17.

5 Rauen T, Eitner F, Fitzner C, Sommerer C, Zeier M, Otte B, et al. Intensive supportive care plus immunosuppression in IgA nephropathy. N Engl J Med. 2015;373(23): 2225-36.

6 Lv J, Zhang H, Wong MG, Jardine MJ, Hladunewich $\mathrm{M}$, Jha $\mathrm{V}$, et al. Effect of oral methylprednisolone on clinical outcomes in patients with IgA nephropathy: the testing randomized clinical trial. JAMA. 2017;318: 432-42.

7 Natale P, Palmer SC, Ruospo M, Saglimbene VM, Craig JC, Vecchio M, et al. Immunosuppressive agents for treating IgA nephropathy. Cochrane Database Syst Rev. 2020;3: CD003965.

8 Suzuki H, Kiryluk K, Novak J, Moldoveanu Z, Herr AB, Renfrow MB, et al. The pathophysiology of IgA nephropathy. J Am Soc Nephrol. 2011;22:1795-803.
9 Moldoveanu Z, Wyatt RJ, Lee JY, Tomana M, Julian BA, Mestecky J, et al. Patients with IgA nephropathy have increased serum galactosedeficient IgA1 levels. Kidney Int. 2007;71: 1148-54.

10 Allen AC, Bailey EM, Brenchley PE, Buck KS, Barratt J, Feehally J. Mesangial IgA1 in IgA nephropathy exhibits aberrant O-glycosylation: observations in three patients. Kidney Int. 2001;60:969-73.

11 Hiki Y, Odani H, Takahashi M, Yasuda Y, Nishimoto A, Iwase H, et al. Mass spectrometry proves under-O-glycosylation of glomerular IgA1 in IgA nephropathy. Kidney Int. 2001;59:1077-85.

12 Zhao N, Hou P, Lv J, Moldoveanu Z, Li Y, Kiryluk K, et al. The level of galactose-deficient IgA1 in the sera of patients with IgA nephropathy is associated with disease progression. Kidney Int. 2012;82:790-6.

13 Gharavi AG, Moldoveanu Z, Wyatt RJ, Barker CV, Woodford SY, Lifton RP, et al. Aberrant IgA1 glycosylation is inherited in familial and sporadic IgA nephropathy. J Am Soc Nephrol. 2008;19:1008-14.

14 Gale DP, Molyneux K, Wimbury D, Higgins P, Levine AP, Caplin B, et al. Galactosylation of IgA1 is associated with common variation in C1GALT1. J Am Soc Nephrol. 2017;28: 2158-66.

15 Gale DP, Molyneux K, Wimbury D, Higgins P, Levine AP, Caplin B, et al. Galactosylation of IgA1 is associated with common variation in C1GALT1. J Am Soc Nephrol. 2017;28: 2158-66.
16 Serino G, Sallustio F, Cox SN, Pesce F, Schena FP. Abnormal miR-148b expression promotes aberrant glycosylation of $\operatorname{IgA} 1$ in $\operatorname{IgA}$ nephropathy. J Am Soc Nephrol. 2012;23: 814-24.

17 Yeo SC, Cheung CK, Barratt J. New insights into the pathogenesis of IgA nephropathy. Pediatr Nephrol. 2018;33:763-77.

18 Rehnberg J, Symreng A, Ludvigsson JF, Emilsson L. Inflammatory bowel disease is more common in patients with IgA nephropathy and predicts progression of ESKD: a Swedish population-based cohort study. J Am Soc Nephrol. 2021;32(2):411-23.

19 Kiryluk K, Li Y, Scolari F, Sanna-Cherchi S, Choi M, Verbitsky M, et al. Discovery of new risk loci for IgA nephropathy implicates genes involved in immunity against intestinal pathogens. Nat Genet. 2014;46:1187-96.

20 McCarthy DD, Kujawa J, Wilson C, Papandile A, Poreci U, Porfilio EA, et al. Mice overexpressing BAFF develop a commensal floradependent, IgA-associated nephropathy. J Clin Invest. 2011;121:3991-4002.

21 Chemouny JM, Gleeson PJ, Abbad L, Lauriero G, Boedec E, Le Roux K, et al. Modulation of the microbiota by oral antibiotics treats immunoglobulin a nephropathy in humanized mice. Nephrol Dial Transplant. 2019;34: 1135-44.

22 De Angelis M, Montemurno E, Piccolo M, Vannini L, Lauriero G, Maranzano V, et al. Microbiota and metabolome associated with immunoglobulin A nephropathy IgAN. PLoS One. 2014;9(6):e99006. 
23 Smerud HK, Fellström B, Hällgren R, Osagie S, Venge P, Kristiánsson G. Gluten sensitivity in patients with IgA nephropathy. Nephrol Dial Transplant. 2009;24:2476-81.

24 Cheung CK, Barratt J. Gluten and IgA nephropathy: you are what you eat? Kidney Int. 2015;88:215-8.

25 Fellström BC, Barratt J, Cook H, Coppo R, Feehally J, de Fijter JW, et al. Targeted-release budesonide versus placebo in patients with IgA nephropathy (NEFIGAN): a doubleblind, randomised, placebo-controlled phase 2b trial. Lancet. 2017;389:2117-27.

26 Barratt J, Rovin BH, Cattran D, Floege J, Lafayette R, Tesar V, et al. Why target the gut to treat IgA nephropathy? Kidney Int Rep. 2020; 5:1620-4.

27 McCarthy DD, Chiu S, Gao Y, Summers-deLuca LE, Gommerman JL. BAFF induces a hyper-IgA syndrome in the intestinal lamina propria concomitant with IgA deposition in the kidney independent of light. Cell Immunol. 2006;241:85-94.

28 Myette JR, Kano T, Suzuki H, Sloan SE, Szretter KJ, Ramakrishnan B, et al. A proliferation inducing ligand (april) targeted antibody is a safe and effective treatment of murine IgA nephropathy. Kidney Int. 2019;96:104-16.

29 Barratt J, Tumlin JA, Suzuki Y, Kao A, Aydemir A, Zima Y, et al. MO039 The 24week interim analysis results of a randomized, double-blind, Placebo-Controlled Phase II Study of atacicept in patients with IGA nephropathy and persistent proteinuria. Nephrol Dial Transplant. 2020;35(Suppl 3).

30 Lafayette RA, Canetta PA, Rovin BH, Appel GB, Novak J, Nath KA, et al. A randomized, controlled trial of rituximab in IgA nephropathy with proteinuria and renal dysfunction. J Am Soc Nephrol. 2017;28:1306-13.

31 Hartono C, Chung M, Perlman AS, Chevalier JM, Serur D, Seshan SV, et al. Bortezomib for reduction of proteinuria in IgA nephropathy. Kidney Int Rep. 2018;3:861-6.

32 Makita Y, Suzuki H, Kano T, Takahata A, Julian BA, Novak J, et al. TLR9 activation induces aberrant IgA glycosylation via APRILand IL-6-mediated pathways in IgA nephropathy. Kidney Int. 2020;97:340-9.

33 Muto M, Manfroi B, Suzuki H, Joh K, Nagai $\mathrm{M}$, Wakai S, et al. Toll-like receptor 9 stimulation induces aberrant expression of a proliferation-inducing ligand by tonsillar germinal center B cells in IgA nephropathy. J Am Soc Nephrol. 2017;28:1227-38.

34 He L, Peng X, Wang J, Tang C, Zhou X, Liu $\mathrm{H}$, et al. Synthetic double-stranded RNA poly(I:C) aggravates IgA nephropathy by triggering IgA class switching recombination through the TLR3-BAFF axis. Am J Nephrol, 2015;42:185-97.

35 Zheng N, Xie K, Ye H, Dong Y, Wang B, Luo $\mathrm{N}$, et al. TLR7 in B cells promotes renal inflammation and Gd-IgA 1 synthesis in IgA nephropathy. JCI Insight. 2020;5.
36 Ciferska H, Honsova E, Lodererova A, Hruskova Z, Neprasova M, Vachek J, et al. Does the renal expression of toll-like receptors play a role in patients with IgA nephropathy? J Nephrol. 2020;33:307-16.

37 Liu LJ, Yang YZ, Shi SF, Bao YF, Yang C, Zhu $\mathrm{SN}$, et al. Effects of hydroxychloroquine on proteinuria in IgA nephropathy: a randomized controlled trial. Am J Kidney Dis. 2019; 74:15-22.

38 Gao W, Xiong Y, Li Q, Yang H. Inhibition of toll-like receptor signaling as a promising therapy for inflammatory diseases: a journey from molecular to nano therapeutics. Front Physiol. 2017;8:508.

39 Novak J, Vu HL, Novak L, Julian BA, Mestecky J, Tomana M. Interactions of human mesangial cells with IgA and IgA-containing immune complexes. Kidney Int. 2002;62: $465-75$.

40 Roberts IS. Pathology of IgA nephropathy. Nat Rev Nephrol. 2014;10(8):445-54.

41 Yanagawa H, Suzuki H, Suzuki Y, Kiryluk K, Gharavi AG, Matsuoka K, et al. A panel of serum biomarkers differentiates IgA nephropathy from other renal diseases. PLoS One. 2014;9:e98081.

42 Maixnerova D, Ling C, Hall S, Reily C, Brown R, Neprasova M, et al. Galactose-deficient IgA1 and the corresponding IgG autoantibodies predict IgA nephropathy progression. PLoS One. 2019;14(2):e0212254.

43 Rizk DV, Saha MK, Hall S, Novak L, Brown R, Huang ZQ, et al. Glomerular immunodeposits of patients with IgA nephropathy are enriched for IgG autoantibodies specific for galactose-deficient IgA1. J Am Soc Nephrol. 2019;30:2017-26.

44 Huang ZQ, Raska M, Stewart TJ, Reily C, King RG, Crossman DK, et al. Somatic mutations modulate autoantibodies against galactose-deficient IgA1 in IgA nephropathy. J Am Soc Nephrol. 2016;27:3278-84.

45 Boyd JK, Barratt J. Immune complex formation in IgA nephropathy: CD89 a "saint" or a "sinner"? kidney. Int. 2010;78:1211-3.

46 Schmitt R, Carlsson F, Mörgelin M, Tati R, Lindahl G, Karpman D. Tissue deposits of IgA-binding streptococcal $\mathrm{M}$ proteins in IgA nephropathy and Henoch-Schonlein purpura. Am J Pathol. 2010;176:608-18.

47 Molyneux K, Wimbury D, Pawluczyk I, Muto M, Bhachu J, Mertens PR, et al. $\beta 1,4-$ galactosyltransferase 1 is a novel receptor for IgA in human mesangial cells. Kidney Int. 2017;92:1458-68.

48 Ma TK, McAdoo SP, Tam FW. Targeting the tyrosine kinase signalling pathways for treatment of immune-mediated glomerulonephritis: from bench to bedside and beyond. Nephrol Dial Transplant. 2017;32:1129-38.

49 Ma TK, McAdoo SP, Tam FW. Spleen tyrosine kinase: a crucial player and potential therapeutic target in renal disease. Nephron. 2016;133:261-9.
50 McAdoo SP, Bhangal G, Page T, Cook HT, Pusey CD, Tam FW. Correlation of disease activity in proliferative glomerulonephritis with glomerular spleen tyrosine kinase expression. Kidney Int. 2015;88:52-60.

51 Kim MJ, McDaid JP, McAdoo SP, Barratt J, Molyneux K, Masuda ES, et al. Spleen tyrosine kinase is important in the production of proinflammatory cytokines and cell proliferation in human mesangial cells following stimulation with IgA1 isolated from IgA nephropathy patients. J Immunol. 2012;189:3751-8.

52 Connell NT, Berliner N. Fostamatinib for the treatment of chronic immune thrombocytopenia. Blood. 2019;133:2027-30.

53 Tam WKF, Tumlin J, Barratt J, Rovin HB, Roberts SDI, Roufosse C, et al. Sun-036 spleen tyrosine kinase (SYK) inhibition in IGA nephropathy: a global, phase II, randomised placebo-controlled trial of fostamatinib. Kidney Int Reports. 2019;4:S168.

54 Sethi S, Fervenza FC. Pathology of renal diseases associated with dysfunction of the alternative pathway of complement: C3 glomerulopathy and atypical hemolytic uremic syndrome (aHUS). Semin Thromb Hemost. 2014;40:416-21.

55 Brilland B, Garnier AS, Chevailler A, Jeannin P, Subra JF, Augusto JF. Complement alternative pathway in ANCA-associated vasculitis: two decades from bench to bedside. Autoimmun Rev. 2020;19:102424.

56 Rizk DV, Maillard N, Julian BA, Knoppova B, Green TJ, Novak J, et al. The emerging role of complement proteins as a target for therapy of IgA nephropathy. Front Immunol. 2019;10: 504

57 Espinosa M, Ortega R, Gómez-Carrasco JM, López-Rubio F, López-Andreu M, López-Oliva MO, et al. Mesangial C4d deposition: a new prognostic factor in IgA nephropathy. Nephrol Dial Transplant. 2009;24:886-91.

58 Roos A, Rastaldi MP, Calvaresi N, Oortwijn $\mathrm{BD}$, Schlagwein N, van Gijlswijk-Janssen DJ, et al. Glomerular activation of the lectin pathway of complement in IgA nephropathy is associated with more severe renal disease. J Am Soc Nephrol. 2006;17:1724-34.

59 Komatsu H, Fujimoto S, Hara S, Sato Y, Yamada K, Eto T. Relationship between serum $\mathrm{IgA} / \mathrm{C} 3$ ratio and progression of $\operatorname{IgA}$ nephropathy. Intern Med. 2004;43:1023-8.

60 Chen P, Yu G, Zhang X, Xie X, Wang J, Shi S, et al. Plasma galactose-deficient IgA1 and C3 and CKD progression in IgA nephropathy. Clin J Am Soc Nephrol. 2019;14:1458-65.

61 Jayne DRW, Merkel PA, Schall TJ, Bekker P. Avacopan for the treatment of ANCA-associated vasculitis. N Engl J Med. 2021;384(7): 599-609.

62 Bruchfeld A, Nachman P, Parikh S, Lafayette R, Potarca A, Diehl J, et al. TO012C5A receptor inhibitor avacopan in IGA Nephropathy Study. Nephrol Dial Transplant. 2017; 32(Suppl 1-3):iii82. 
63 Selvaskandan H, Kay Cheung C, Dormer J, Wimbury D, Martinez M, Xu G, et al. Inhibition of the lectin pathway of the complement system as a novel approach in the management of IgA vasculitis-associated nephritis. Nephron. 2020;144:453-8.

64 Lafayette RA, Rovin BH, Reich HN, Tumlin JA, Floege J, Barratt J. Safety, tolerability and efficacy of narsoplimab, a novel MASP-2 inhibitor for the treatment of IgA nephropathy. Kidney Int Rep. 2020;5(11):2032-41.

65 Trachtman H, Nelson P, Adler S, Campbell KN, Chaudhuri A, Derebail VK, et al. DUET: a Phase 2 Study evaluating the efficacy and safety of sparsentan in patients with FSGS. J Am Soc Nephrol. 2018;29:2745-54.

66 Heerspink HJL, Stefánsson BV, Correa-Rotter R, Chertow GM, Greene T, Hou FF, et al. Dapagliflozin in patients with chronic kidney disease. N Engl J Med. 2020;383:1436-46.
67 Wheeler DC, Toto RD, Stefansson BV, Jongs $\mathrm{N}$, Chertow GM, Greene T, et al. A pre-specified analysis of the DAPA-CKD trial demonstrates the effects of dapagliflozin on major adverse kidney events in patients with IgA nephropathy. Kidney Int. 2021;100(1):215-24.

68 Barratt J, Floege J. SGLT-2 inhibition in IgA nephropathy: the new standard-of-care? Kidney Int. 2021;100(1):24-6.

69 Herrington WG, Preiss D, Haynes R, Von Eynatten $\mathrm{M}$, Staplin N, Hauske SJ, et al. The potential for improving cardio-renal outcomes by sodium-glucose co-transporter-2 inhibition in people with chronic kidney disease: a rationale for the EMPA-KIDNEY study. Clin Kidney J. 2018;11:749-61.

70 Lechner SM, Abbad L, Boedec E, Papista C, Le Stang MB, Moal C, et al. IgA1 protease treatment reverses mesangial deposits and hematuria in a model of IgA nephropathy. J Am Soc Nephrol. 2016;27:2622-9.
71 Wang L, Li X, Shen H, Mao N, Wang H, Cui $\mathrm{L}$, et al. Bacterial IgA protease-mediated degradation of agIgA1 and agIgA 1 immune complexes as a potential therapy for IgA nephropathy. Sci Rep. 2016;6:30964-13.

72 Jordan SC, Lorant T, Choi J, Kjellman C, Winstedt L, Bengtsson M, et al. IgG endopeptidase in highly sensitized patients undergoing transplantation. N Engl J Med. 2017;377: $442-53$.

73 Soveri I, Mölne J, Uhlin F, Nilsson T, Kjellman C, Sonesson E, et al. The IgG-degrading enzyme of Streptococcus pyogenes causes rapid clearance of anti-glomerular basement membrane antibodies in patients with refractory anti-glomerular basement membrane disease. Kidney Int. 2019;96:1234-8. 